\title{
Convergence of day-to-day traffic flow dynamics under tradable bottleneck permits
}

\author{
K. Wada ${ }^{1}$, T. Akamatsu ${ }^{1} \&$ S. Kikuchi ${ }^{2}$ \\ ${ }^{I}$ Graduate School of Information Sciences, Tohoku University, Japan \\ ${ }^{2}$ Ministry of Land, Infrastructure, Transport and Tourism, Japan
}

\begin{abstract}
Previously, Akamatsu et al. proposed "tradable bottleneck permits" as a new transportation demand management scheme and proved the efficiency thereof for a general network. To implement such a scheme, we propose a multi-agent system for general transportation networks. The aim of this system is to achieve a socially optimal state in which the total transportation cost is minimized by the decentralized behaviour of agents. As a concrete step in designing the proposed system, we first define the micro behaviour of the agents. We also assume that the trading markets for bottleneck permits are described by a tâtonnement process. We then derive day-to-day dynamics of aggregated traffic flows and permit prices. By analyzing the macro dynamics, we prove that the mean dynamics of the aggregated variables (flows and permit prices) converge to a socially optimal state.
\end{abstract}

Keywords: tradable bottleneck permits, multi-agent system, evolutionary game theory.

\section{Introduction}

Before new road regulations are implemented, the road manager is normally required to have accurate information on the users' behaviour (i.e., precise demands). For instance, in standard congestion pricing (see, for example Yang and Huang [1]), the manager needs to know the potential number of users, their desired arrival times and their value of time. It is, however, not always possible for such private information to be obtained completely and accurately. If the regulations are implemented with incomplete information, this will inevitably 
result in an economic loss. An important point to emphasize here is that the problem lies in the asymmetry of information between the road manager and users.

A transportation demand management (TDM) scheme that resolves the asymmetric information problem is "tradable bottleneck permits" (TBP) proposed by Akamatsu et al. [2], Akamatsu [3]. This scheme is based on the following: a) the road manager issues a right that allows the permit holder to pass through the bottleneck during a pre-specified time period, and b) a new trading market is established for bottleneck permits differentiated by the prespecified time. In this scheme, the road manager only needs to know the traffic capacity of each link (as queuing congestion can be eliminated merely by issuing a number of permits for each link equal to or less than the link capacity). It has been proved that equilibrium resource allocation under the scheme is efficient in the sense that the total transportation cost in the network is minimized for general networks.

Although the TBP has the theoretically-desirable properties mentioned above, the following problems still need to be addressed: $i$ ) the processes for achieving equilibrium traffic flow patterns are not shown, and $i$ ) the procedures for trading bottleneck permits are cumbersome. The first problem requires a framework that can describe the processes, while the latter problem requires a system that alleviates the cumbersome procedure.

To deal with these problems, we propose a multi-agent system for implementing the TBP in general transportation networks. In such a system, vehicle-installed agent software chooses, on behalf of the user, an optimal path and arrival/departure times based on the user's preferences. Each agent further deals with the cumbersome procedure of trading the bottleneck permits. The system is required to have the following properties: $i$ ) each agent can choose a path and an arrival time using local information only (autonomy of behaviour), $i i$ ) the algorithm for the agent's behaviour is simple (briefness of agent behaviour rules), and iii) the whole system can converge to equilibrium (stability of dynamics).

The purpose of this paper is to design the essential parts of the multi-agent system to achieve a socially optimal state under the TBP. As a concrete step in designing the proposed system, we first define the micro behaviour of the agents. Specifically, for the agents' behaviour, we use the perturbed best response model that is popular in evolutionary game theory. We also assume that the dynamics of the price of bottleneck permits in the trading market is described by tâtonnement dynamics. We then derive the day-to-day dynamics of aggregated traffic flows and permit prices. By analyzing the macro dynamics, we prove that the mean dynamics of the aggregated variables (flows and permit prices) converges to a socially optimal state.

\section{The model}

\subsection{Networks}

In this paper, we consider dynamic traffic flows in a network $G$ that consists of a freeway network and a city street network. The freeway network is located 
upstream and the city street network downstream, and these networks are connected by off-ramp nodes. In the freeway network, we assume that queuing congestion occurs, but flow congestion does not. In the city street network, on the other hand, we assume that flow congestion occurs, but queuing congestion does not.

The network $G$ consists of a set of nodes $N$, and a set of directed links $L$. The node set $N$ includes an origin node being the start of a user's trip, and a destination node at which the trip is terminated. Each element of $N$ (i.e., each node) is identified by a sequential natural number $i$, and each element of $L$ (i.e., each link) is denoted by a pair $(i, j)$ consisting of the upstream node $i$ and the downstream node $j$. Each origin-destination (OD) pair is denoted by the pair ( $o$, d) and $W$ denotes a set of OD pairs.

We also assume that each link $(i, j)$ in the freeway network has a bottleneck that is represented by a point queue model with constant capacity $\mu^{i j}$. The travel time to pass through the link $(i, j)$ in the freeway network is constant $c^{i j}$, since there is no flow congestion. In the city street network, on the other hand, the travel time to use the link $(i, j)$ is given by a strictly monotone function $c^{i j}\left(y^{i j}\right)$ : $\partial c^{i j}\left(y^{i j}\right) / \partial y^{i j}>0$ where $y^{i j}$ is the traffic flow per unit of time. We assume that the travel demand $Q$, with respect to trips per day, is a given constant.

We consider time-dependant traffic flow patterns within daily and day-to-day traffic flow dynamics. We then distinguish the day $t \in T$ from the time $s \in S$ within day $t$. Furthermore, the day $t$ and time $s$ are also identified by sequential natural numbers.

Each user makes a single trip per day from an origin (e.g., residential zone) to a destination (e.g., CBD) in the network. Each user chooses a destination arrival time and a path between the origin and destination so as to minimize his or her generalized transportation cost.

\subsection{TDM schemes}

The TBP can completely eliminate queuing congestion, but cannot eliminate flow congestion. We consider a hybrid scheme that combines the TBP with congestion pricing. We also consider the congestion pricing proposed by Sandholm [4] to alleviate flow congestion.

\subsubsection{Tradable bottleneck permits}

A road manager issues time-dependant bottleneck permits for all bottlenecks (i.e., links) in the freeway network to eliminate queuing congestion. We assume that the number of permits issued for each link for each time unit is equal to or less than the traffic capacity of that link in the network. This means that queuing congestion never occurs in the network under the TBP scheme.

The permits issued for each link (bottleneck) are offered for sale by the road manager. There are as many markets for trading permits as there are links, and each market is dedicated to trading the permits for that link. We also assume that the trading markets are perfectly competitive; that is, each agent acts as a pricetaker. 


\subsubsection{Congestion pricing}

A road manager imposes congestion tolls on the users for all links in the city street network to alleviate flow congestion. In standard congestion pricing, however, the road manager cannot determine appropriate tolls without having accurate user information. We thus consider the congestion pricing proposed by Sandholm [4]. The method of implementing this model is evolutionary in that congestion tolls are determined solely from temporal traffic flows. We refer to this scheme as "evolutionary congestion pricing" and give a precise definition thereof in Subsection 4.2 below.

\subsubsection{Multi-agent system}

To implement the hybrid scheme we consider a multi-agent system, in which vehicle-installed agent software chooses, on behalf of a user, an optimal path and arrival/departure times according to the user's preferences, so as to minimize the generalized transportation cost. Each agent further deals with the cumbersome procedure in the trading markets for bottleneck permits.

\section{Socially optimal state}

This section defines the socially optimal state under the TBP with evolutionary congestion pricing, and shows the relationships between socially optimal and static equilibrium conditions.

\subsection{Dynamic socially optimal assignment problem}

In order to define the socially optimal state, consider the following optimization problem [P-1]:

$$
\min _{(\mathbf{q}, y) \in \Omega} \gamma \sum_{s \in S} \sum_{i j \in L} y_{s}^{i j} c^{i j}\left(y_{s}^{i j}\right)+\sum_{o d \in W \in S} \sum_{s \in S} q_{s}^{o d} d_{s}
$$

subject to

$$
\begin{array}{cc}
\sum_{s \in S} q_{s}^{o d}=Q^{o d} & \forall o d \in W \\
y_{s}^{i j}=\sum_{o \in O} y_{s}^{i j, o} & \forall i j \in L, \forall s \in S \\
\sum_{j \in N O(k)} y_{s}^{k j, o}-\sum_{i \in N(k)} z_{s}^{i k}=-q_{s}^{o d} \delta_{k, d} & \forall k \in N, \forall o \in O, \forall s \in S \\
y_{s}^{i j, o}=z_{\tau}^{i j, o} & \forall i j \in L, \forall o \in O, \forall s \in S \\
y_{s}^{i j} \leq \mu^{i j} & \forall i j \in L, \forall s \in S \\
y_{s}^{i j} \geq 0 & \forall i j \in L, \forall s \in S
\end{array}
$$

where $q_{s}^{o d}$ is the OD traffic flow rate for arriving at the destination at time $s, y_{s}^{i j}$ is the traffic flow rate for arriving at link $(i, j)$ at time $s, \gamma$ is a coefficient that converts travel time to a monetary equivalent, and $d_{s}$ is the schedule cost of arriving at the destination at time $s$. Furthermore, $\Omega$ is a feasible domain that 
satisfies Eqs. (2)-(7). Equation (2) denotes flow conservation for OD flow rates and OD travel demands $Q^{\text {od }}$. Equation (3) denotes flow conservation for link flows and link inflows from each origin. The constraints in (4) denote flow conservation at each node; that is, the conservation of the dynamic traffic flow in a network is represented as the equality of inflows and outflows at each node for each point in time. To formalize this, we let $z_{s}^{i j, o}$ be the flow rate departing from link $(i, j)$ at time $s$ from the origin $o . \quad \delta_{k, d}$ denotes Kronecker's delta (i.e., 1 if $k$ $=d$, zero otherwise); $N O(k)$ is the set of downstream nodes of the links incident to node $k ; \mathrm{NI}(\mathrm{k})$ is the set of upstream nodes of the links incident to node $k$. Equation (5) denotes the First-In-First-Out conditions on each link. We assume that dynamic traffic flow in our model satisfies the First-In-First-Out property on each link (i.e., we assume that overtaking can be ignored). $\tau$ denotes the departure time of link $(i, j)$ for a user entering the link at time $s$ :

$$
\tau=s+c^{i j}\left(y_{s}^{i j}\right)
$$

Constraint (6) is the capacity constraint on each link and constraint (7) is the nonnegative constraint.

\subsection{Relationships between the social optimum and static equilibrium}

We show that the socially optimal state is equivalent to the static equilibrium under the TBP with evolutionary congestion pricing. Specifically, the optimal conditions for [P-1] are equivalent to Eqs. (2)-(8) and the static equilibrium conditions given below (proof: see Akamatsu [3]).

\section{1) Equilibrium conditions for the path choice:}

At equilibrium, no user can improve his or her own cost by changing the path choice unilaterally. To formalize this, let $\pi_{s}^{o i}$ be the minimal travel cost to node $i$ from origin $o$, and $u_{s}^{i j}$ be the transportation cost on link $(i, j)$ :

$$
u_{s}^{i j} \equiv p_{s}^{i j}+\lambda_{s}^{i j}\left(y_{s}^{i j}\right)+c^{i j}\left(y_{s}^{i j}\right)
$$

where $p_{s}^{i j}$ is the price of a permit for link $(i, j)$ at the specified time $s$, and $\lambda_{s}^{i j}$ is the congestion toll. Then the equilibrium condition is represented as

$$
\left\{\begin{array}{l}
\pi_{\tau}^{o j}=u_{s}^{i j}+\pi_{s}^{o i} \text { if } y_{s}^{i j}>0 \\
\pi_{\tau}^{o j} \leq u_{s}^{i j}+\pi_{s}^{o i} \text { if } y_{s}^{i j}=0
\end{array} \quad \forall i j \in L, \forall o \in O, \forall s \in S\right.
$$

\section{2) Equilibrium conditions for destination arrival time choice:}

At equilibrium, no one can improve his or her own generalized transportation cost by changing the destination arrival time unilaterally. The generalized transportation cost for a user arriving at time $s$ is $\pi_{s}^{o d}+d_{s}$. Therefore, the equilibrium condition for the user's arrival time choice can be expressed as

$$
\left\{\begin{array}{l}
\rho=\pi_{s}^{o d}+d_{s} \text { if } q_{s}^{o d}>0 \\
\rho \leq \pi_{s}^{\text {od }}+d_{s} \text { if } q_{s}^{\text {od }}=0
\end{array} \quad \forall o d \in W, \forall s \in S\right.
$$

where $\rho$ represents the minimum (equilibrium) generalized transportation cost. 


\section{3) Demand-supply equilibrium (market clearing) conditions in each link permit market:}

At equilibrium, if the price of a certain type of permit is positive, the quantity supplied equals the quantity demanded for that permit; for a permit whose supply quantity exceeds the quantity demanded, the price is zero. Note that, for each link $(i, j)$ and each time $s$, the demand for a permit of that link at time $s$ is equal to the inflow rate $y_{s}^{i j}$.

$$
\left\{\begin{array}{l}
y_{s}^{i j}=\mu^{i j} \quad \text { if } p_{s}^{i j}>0 \\
y_{s}^{i j} \leq \mu^{i j} \quad \text { if } p_{s}^{i j}=0
\end{array} \quad \forall i j \in L, s \in S\right.
$$

The socially optimal state (or its equivalent, the equilibrium state) is defined as described above, but the processes for achieving the socially optimal state have not yet been clarified. In the following sections, we consider the day-to-day dynamics of traffic flows and prices, and show that the multi-agent system can achieve the socially optimal state under the proposed scheme.

\section{Price dynamics under the proposed scheme}

\subsection{Price dynamics in the permit trading markets}

We assume that the dynamics of the price of bottleneck permits in the trading markets is described by tâtonnement dynamics. The price of a permit on day $t$ is adjusted so as to clear the excess demand for that type of permit.

Let $D_{s}^{i j}$ be the demand for a permit for link $(i, j)$ at time $s$. On the other hand, the supply (upper bound) of a permit for link $(i, j)$ at time $s$ is a constant $\mu^{i j}$. Thus the excess demand on day $t$ is denoted by $\left(D_{s}^{i j}(t)-\mu^{i j}\right)$. Then the permit price $p_{s}^{i j}(t)$ is adjusted as follows:

$$
p_{s}^{i j}(t+1)=\max \cdot\left[\left(D_{s}^{i j}(t)-\mu^{i j}\right) \cdot K+p_{s}^{i j}(t), 0\right]
$$

where $K$ is a positive constant.

\subsection{Evolutionary congestion pricing}

To alleviate flow congestion, we introduce "evolutionary congestion pricing" proposed by Sandholm [4]. The method of implementation is evolutionary, requiring that the road manager sets congestion tolls using temporal information (i.e., the traffic flow pattern that occurs on day $t$ ) as follows:

$$
\lambda_{s}^{i j}(t+1)=\gamma \cdot y_{s}^{i j}(t) \cdot \partial c^{i j}\left(y_{s}^{i j}(t)\right) / \partial y_{s}^{i j}
$$

In his study, Sandholm [4] considered traffic flows in a network with flow congestion and proved that the traffic flow pattern under the scheme eventually converges to a socially optimal state. The micro behaviour of each agent was, however, not clarified. We, therefore, consider an agent's micro behaviour under evolutionary congestion pricing, and derive the day-to-day dynamics of traffic flows and prices. 


\section{Agents' behaviour}

We consider that each agent plays a game (i.e., the trip) on each day $t$. Let the combination of path and destination arrival time $\{r, s\}$ be the strategy of the agent, and $\{R(o, d), S\}$ be the set of strategies available to the agents including the OD pair. The payoff (i.e., generalized transportation cost) of the strategy $\{r$, $s$ \} depends on the total number of agents choosing the strategy.

\subsection{Perturbed best response}

For the agents' behaviour, we consider the perturbed best response model which is a rule referring to the traffic flow pattern that occurred on day $t$. In this case, the road manager only counts the traffic flows that occurred on day $t$, and informs the agents about this data.

In the perturbed best response model that is popular in evolutionary game theory, each agent makes his choices after the payoffs have been subjected to random shocks. The payoff of strategy $r(o, d)$ is defined as the sum of the link costs included on path $r(o, d)$ :

$$
U^{r(o, d)}(t) \equiv-\sum_{i j \in L} u_{s}^{i j}(t) \cdot \delta_{i j, r(o, d)}
$$

where $\delta_{i j, r(o, d)}$ is a typical element of the path-link incidence matrix for the node pair $(o, d)$ : the value is 1 if link $(i, j)$ is on the path $r$ connecting the OD pair $(o$, $d$ ); otherwise, it is zero. Each agent chooses a strategy so as to maximize the payoff defined as Eq. (15), adding to the random disturbance term.

We obtain the strategy by solving a two-stage (hierarchical) choice problem, in which the destination arrival time $s$ (the upper-level choice) and the path $r$ (the lower-level choice) are chosen separately. More specifically, agent $\alpha$ first solves the lower-level problem of the path choice for a given arrival time $s$; he then obtains the route choice $r_{s}^{\alpha}(t+1)$ and the maximum value $\pi_{s}^{\alpha}(t)$ condition on arrival time $s$ :

$$
\begin{aligned}
& -\pi_{s}^{\alpha}(t)=\max _{r \in R(o, d)} U_{s}^{r(o, d)}(t)+\xi_{r(o, d)}^{\alpha}(t) \\
& r_{s}^{\alpha}(t+1)=\underset{r \in R(o, d)}{\arg \cdot \max } \cdot U_{s}^{r(o, d)}(t)+\xi_{r(o, d)}^{\alpha}(t)
\end{aligned}
$$

By using this maximum choice function, the upper-level problem of the arrival time choice is reduced to

$$
s^{\alpha}(t+1)=\underset{s \in S}{\arg . \max } \cdot-\pi_{s}^{\alpha}(t)-d_{s}+\varepsilon_{s}^{\alpha}(t)
$$

where $\xi_{r(o, d)}^{\alpha}$ and $\varepsilon_{s}^{\alpha}$ are random disturbance terms: each agent chooses a mixed strategy on each day $t$.

Furthermore, we assume that $\xi_{r(o, d)}^{\alpha}$ and $\varepsilon_{s}^{\alpha}$ are i.i.d. with the Gumbel distribution. According to a random utility model, the choice probability $l_{s}^{r(o, d)}$ of the path and the choice probability $h_{s}$ of the arrival time are formulated as the following nested logit model: 


$$
\begin{aligned}
& l_{s}^{r(o, d)}(t+1)=\exp \left[\theta U^{r(o, d)}(t)\right] / \sum_{\widetilde{r} \in R(o, d)} \exp \left[\theta U^{\widetilde{r}(o, d)}(t)\right] \\
& h_{s}(t+1)=\exp \left[-\eta\left(\pi_{s}^{o d}(t)+d_{s}\right)\right] / \sum_{\widetilde{s} \in S} \exp \left[-\eta\left(\pi_{\widetilde{s}}^{o d}(t)+d_{\widetilde{s}}\right)\right]
\end{aligned}
$$

where $\pi_{s}^{o d}(t)$ is the expected utility of the path choice, and $\theta$ and $\eta$ are logit parameters. Using these choice probabilities, the choice probability of agent $\alpha$ 's strategy $b_{s}^{r(o, d)}$ is denoted by

$$
b_{s}^{r(o, d)}(t)=l_{s}^{r(o, d)}(t) \cdot h_{s}(t)
$$

This means that the agents of the same OD pair have the same choice probability.

When each agent chooses a strategy according to the rules given above, there is the possibility that the demand of link $(i, j)$ at time $s$ exceeds the supply $\mu^{i j}$ : the permit is sold out. In that case, the road manager assigns the permits to the agents according to a rule proposed by Kikuchi et al [5]. By following these rules, all agents eventually select strategies, and all the capacity constraints are met.

\section{Convergence of day-to-day dynamics}

\subsection{Expected changes in day-to-day dynamics}

In this section, we consider the day-to-day dynamics, for each arrival time, of a path flow $f_{s}^{r(o, d)}(t)$ and a permit price $p_{s}^{i j}(t)$. These dynamics are stochastic, so the first step in their analyses is to determine their expected motion.

Let $\mathbf{X}(t) \equiv[\mathbf{f}(t) \mathbf{p}(t)]^{T}$ denote the combination of the path flow and the permit price. If $\mathbf{X}(t)$ is a state $\mathbf{X} \equiv[\mathbf{f} \mathbf{p}]^{T}$, the payoff $\mathbf{U}$ and the choice probability $\mathbf{b}$ are denoted by $\mathbf{U}(t)=\mathbf{U}(\mathbf{x}), \mathbf{b}(t)=\mathbf{b}(\mathbf{x})$. In this case, the path flow can be described by

$$
E[\mathbf{f}(t+1)-\mathbf{f}(t) \mid \mathbf{X}(t)=\mathbf{x}]=\hat{\mathbf{F}}(\mathbf{x})-\mathbf{f}
$$

where $\hat{F}_{s}^{r(o, d)}(\mathbf{x})$ is the expected number of agents who choose strategy $\{r, s\}$ in state $\mathbf{x}$.

When the road manager assigns the permits to the agents according to the rule proposed by Kikuchi and Akamatsu [5], the expected number of agents choosing strategy $\{r, s\}$ is determined by solving the following optimization problem [P-2]:

$$
\hat{\mathbf{F}}(\mathbf{x})=\arg \max _{\hat{\mathbf{f}} \geq \mathbf{0}} \cdot \mathbf{U}^{T}(\mathbf{x}) \hat{\mathbf{f}}-\hat{\mathbf{q}}^{T}(\hat{\mathbf{f}}) \mathbf{v}_{\mathbf{L}}(\hat{\mathbf{f}})-\mathbf{Q}^{T} \mathbf{v}_{\mathbf{H}}(\hat{\mathbf{f}})
$$

subject to $i$ ) flow conservation for OD flow rates and OD travel demands, $i i$ ) flow conservation for path flows and OD flows, and iii) capacity constraints on each link. $\mathbf{v}_{\mathbf{L}}$ and $\mathbf{v}_{\mathbf{H}}$ denote the entropy terms for the lower-level choice and upper-level choice, respectively: 


$$
\begin{gathered}
\left(v_{L}\right)_{s}^{o d}(\mathbf{F})=\frac{1}{\theta} \sum_{r \in R(o, d)}\left(F_{s}^{r(o, d)} / \hat{q}_{s}^{o d}\right) \ln \left(F_{s}^{r(o, d)} / \hat{q}_{s}^{o d}\right) \\
\left(v_{H}\right)^{o d}(\mathbf{F})=\frac{1}{\eta} \sum_{s \in S}\left(\hat{q}_{s}^{o d} / Q^{o d}\right) \ln \left(\hat{q}_{s}^{o d} / Q^{o d}\right)
\end{gathered}
$$

If a link capacity constraint is ineffective, $\hat{\mathbf{F}}$ is equivalent to the following $\mathbf{B}$ :

$$
B_{s}^{r(o, d)}(\mathbf{x})=Q^{o d} \cdot b_{s}^{r(o, d)}(\mathbf{x})
$$

In contrast to Eq. (13), the expected change in the permit price is denoted by a function that is governed only by the state $\mathbf{x}$ :

$$
E[\mathbf{p}(t+1)-\mathbf{p}(t) \mid \mathbf{X}(t)=\mathbf{x}]=\max \cdot[(\hat{\mathbf{D}}(\mathbf{x})-\mu) \cdot K,-\mathbf{p}]
$$

where $\hat{D}^{i j}(\mathbf{x})$ is the expected demand for a permit on link $(i, j)$ computed by traffic flows and permit prices on day $t$ :

$$
\hat{D}_{s}^{i j}(\mathbf{x})=\sum_{o d \in W} \sum_{r \in R(o, d)} B_{s}^{r(o, d)}(\mathbf{x}) \cdot \delta_{i j, r(o, d)}
$$

In order to discuss convergence of the dynamics, we derive continuous time version expected changes corresponding to the discrete time expression of Eqs. (22) and (27). We assume that the frequency of changing strategies by each agent is the same and constant. Under these assumptions, the expected change of $\mathbf{X}(t)$ can be described by a continuous time Markov process:

$$
E[\dot{\mathbf{X}}(t) \mid \mathbf{X}(t)=\mathbf{x}]=\left[\begin{array}{c}
\kappa_{f} \cdot\{\hat{\mathbf{F}}(\mathbf{x})-\mathbf{f}\} \\
\kappa_{p} \cdot \mathbf{H}(\mathbf{x})
\end{array}\right]
$$

where $\kappa_{f}$ and $\kappa_{p}$ are positive constants, and $H^{i j}$ is the function

$$
H_{s}^{i j}(\mathbf{x})=\max \left[\hat{D}_{s}^{i j}(\mathbf{x})-\mu^{i j}, 0\right]
$$

\subsection{Properties of the dynamics}

From the above, we obtain the following proposition for a rest point defined as $E\left[\dot{\mathbf{X}}(t) \mid \mathbf{X}(t)=\mathbf{x}^{*}\right]=\mathbf{0}$ :

Proposition 1: Consider the Markov process denoted by Eq. (29). If the parameter $\theta$ approaches infinity, a rest point $\mathbf{x}^{*}$ is equivalent to the resource allocation in the sense that the total transportation cost in the network is minimized as denoted by problem [P-1]. (Proof: see Kikuchi and Akamatsu [5])

On the surface, if $\mathbf{X}(t)$ converges, this proposition states that equilibrium traffic patterns coincide with the socially optimal state.

To state our convergence result, we consider a continuous differentiable function as follows:

$$
\begin{aligned}
\Pi(\mathbf{x}) \equiv-\mathbf{U}^{T}(\mathbf{x}) \mathbf{f}+\hat{\mathbf{q}}^{T}(\mathbf{f})\left(\mathbf{v}_{\mathbf{L}}(\mathbf{f})+\mathbf{w}_{\mathbf{L}}(\mathbf{x})\right) \\
+\mathbf{Q}^{T}\left(\mathbf{v}_{\mathbf{H}}(\mathbf{f})+\mathbf{w}_{\mathbf{H}}(\mathbf{x})\right)+\frac{1}{2}(-\hat{\mathbf{D}}(\mathbf{x}))^{T}(-\hat{\mathbf{D}}(\mathbf{x}))
\end{aligned}
$$

where

$$
\left(w_{L}\right)_{s}^{o d}(\mathbf{x})=\frac{1}{\theta} \ln \sum_{r \in R(o, d)} \exp \left[\theta U_{s}^{r(o, d)}(\mathbf{x})\right]
$$




$$
\left(w_{H}\right)^{\text {od }}(\mathbf{x})=\frac{1}{\eta} \ln \sum_{s \in S} \exp \left[-\eta\left(\pi_{s}^{\text {od }}(\mathbf{x})+d_{s}\right)\right]
$$

A function that is $\Pi(\mathbf{x})$ added to a constant value is a strict Lyapunov function for dynamic (29) (see the detailed proof by Kikuchi and Akamatsu [5]). By stochastic approximation theory (see, Benaïm [6]), we obtain the following proposition:

Proposition 2: Under the rule of the agents' behaviour defined in Section 5, the dynamics of the path flow $\mathbf{f}(t)$ and the permit price $\mathbf{p}(t)$ converge globally.

The discussion above shows that, under the rule of the agents' behaviour in this paper, the traffic patterns can globally converge and the equilibrium resource allocation is equivalent to a socially optimal state.

\section{Conclusion}

In this paper, we proposed a multi-agent system for implementing tradable bottleneck permits. The aim of such a system is to achieve a socially optimal state in which the total transportation cost is minimized through decentralized behaviour of the agents. As a concrete step in designing the proposed system, we first defined the micro behaviour of the agents. We then derived day-to-day dynamics of aggregate variables (flows and permit prices) from the micro behaviour. By analyzing the macro dynamics, we proved that the mean dynamics of aggregate variables globally converge to a socially optimal state.

\section{References}

[1] Yang, H. \& Huang, H.J., Mathematical and Economic Theory of Road Pricing, Elsevier Science \& Technology, Oxford, UK, 2005.

[2] Akamatsu, T., Sato, S. \& Nguyen, X.L., Tradable time-of-day bottleneck permits for morning commuters. JSCE Journal of Infrastructure Planning and Management 62, pp. 605-620, 2006.

[3] Akamatsu, T., Tradable Network Permits: A New Scheme for the Most Efficient Use of Network Capacity. Submitted to Transportation Science.

[4] Sandholm, W.H., Evolutionary Implementation and Congestion Pricing. Review of Economic Studies, pp.667-689, 2002.

[5] Kikuchi, S. \& Akamatsu, T., Dynamics of Decentralized Multi-Agent Systems for Imple-menting Tradable Network Permits, Infrastructure Planning Review, in press.

[6] Benaïm, M., Recursive Algorithms, Urn Processes, and Chaining Number of Chain Recurrent Sets, Ergodic Theory and Dynamical Systems, 18, pp.5387, 1998. 